\title{
ENSACAMENTO DE FRUTOS DE PEREIRA CV. HOUSUI ${ }^{1}$
}

\author{
IVAN DAGOBERTO FAORO² \& MARCIA MONDARDO 3
}

RESUMO - Foi avaliada a qualidade de frutos de pêra japonesa cv. Housui (Pyrus pyrifolia var. culta) ensacados com diferentes tipos de sacos de papel e em duas épocas: 34 e 83 dias após a florada. O ensacamento não influenciou na firmeza, no teor de sólidos solúveis totais e no peso médio dos frutos. Sacos vermelhos não induziram aumento do $\mathrm{pH}$ da polpa, mas o uso de sacos duplos, sacos marrons, sacos de papel kraft marrons e sacos de pipoca brancos aumentaram significativamente o $\mathrm{pH}$. Ao buscar-se maior precocidade de colheita, os melhores resultados foram obtidos com o uso de sacos pequenos de papel manteiga aos 34 dias após a floração e o uso de sacos grandes duplos ou sacos grandes marrons, 83 dias após a plena floração. O ensacamento 34 dias após a plena floração, com sacos grandes de papel duplo de cor marrom ou sacos de papel kraft marrons, ou ainda o uso de sacos pequenos parafinados transparentes de papel manteiga, aos 34 dias, seguidos pela colocação, aos 83 dias, dos dois tipos de sacos grandes citados anteriormente, resultaram em frutos de melhor qualidade externa (película de coloração homogênea e mais clara, lisa e com lenticelas pouco salientes). O uso de sacos vermelhos de papel manteiga e de sacos de pipoca brancos, com ou sem ensacamento prévio com sacos pequenos de papel manteiga parafinado, não resultaram em melhoria substancial da qualidade externa do fruto.

Termos para indexação: Pyrus, pêra japonesa, ensacamento, qualidade do fruto.

\section{BAGGING OF NASHI PEAR CV. HOUSUI}

ABSTRACT - The fruit quality of Nashi pears cv. Housui (Pyrus pyrifolia var. culta) have been evaluated after bagging with different paper bags at two bagging dates: 34 and 83 days after full bloom. The bagging did not affect the fruit firmness, the total soluble solids and the average fruit weight. Red bags did not affect the $\mathrm{pH}$ of the fruit flesh. However the fruit bagged with double bags of brown color, brown kraft paper and white popcorn paper increased the $\mathrm{pH}$. In order to harvest precociously, the use of small transparent paraffin paper bags 34 days after full bloom or the use of large kraft paper bags of brown color and also by using small transparent paraffin paper bags at 34 days followed by the use of a double large bags at 83 days after full bloom, resulted in better fruit appearance (uniform, shine and smooth skin color with small lenticels). The use of red bags and white popcorn bags, with and without previous bagging with small transparent paraffin paper bags did not result on better fruit appearance.

Index terms: Pyrus, Nashi pears, bagging, fruit quality.

\section{INTRODUÇÃO}

A pêra japonesa é ainda pouco conhecida pela maioria dos consumidores e produtores brasileiros, por ser recente o seu cultivo em escala comercial. Aliado a isto, outro fator que reduz o seu consumo é o alto preço dos frutos, razão pela qual são direcionados para nichos de mercado de consumidores com maior poder aquisitivo.

Esta cultura surge com possibilidade de ser produzida sem o uso de agrotóxicos, desde que suas frutas sejam protegidas e não ocorra excesso de chuva no período produtivo (Faoro, 2003). Pesquisa conduzida em Caçador-SC mostrou que a 'Housui' é moderadamente resistente à entomosporiose (Diplocarpon mespilli) (Faoro et al., 2001). Observações a campo indicam que a 'Housui' também é resistente à seca dos ramos (Botryosphaeria sp.). No entanto, todas as peras são facilmente atacadas pela mosca-das-frutas (Anastrepha fraterculus) e pela mariposa oriental (Grapholita molesta) (Nora \& Sugiura, 2001). Mas os danos de doenças e pragas têm a incidência reduzida para $1,1 \%$ a 3,8 \% quando os frutos são ensacados (Faoro \& Yasunobu, 2001). Isso motivou o desenvolvimento desta pesquisa, aliado à inexistência de informações no Brasil sobre o efeito do ensacamento em pêra japonesa. Desta forma, o objetivo deste trabalho foi testar o efeito de diferentes tipos de sacos de papel na qualidade de frutos de pereira cv. Housui.

\section{MATERIALEMÉTODOS}

O experimento foi realizado na Epagri, Estação Experimental de Caçador, em Caçador-SC, entre 30-10-2000 e 1º-02-2001, utilizando a cultivar de pereira japonesa Housui (Pyrus pyrifolia var. culta). Os tratamentos estão relacionados na Tabela 1 .

Os sacos pequenos, utilizados no ensacamento aos 34 dias após a plena floração, eram de papel manteiga parafinado e transparente $(6,3 \times 7,0 \mathrm{~cm})$. Os sacos vermelhos (papel manteiga com 16,5 x 19,5 cm), marrons (papel manteiga com 14,2 x 17,8 cm) e duplos (saco interno de papel manteiga de cor amarela e saco externo de papel manteiga de cor marrom com 15,0 x 17,0 cm) foram fabricados no Japão, onde são usualmente utilizados no ensacamento de pêra. Os sacos de papel pipoca brancos (13,0 x 20,0) e o saco de papel marrom Kraft comercial (13,5 x 20,7 $\mathrm{cm}$ ) foram obtidos no Brasil, em lojas comerciais.

TABELA 1-Época de realização e tipos de sacos utilizados no ensacamento de peras da cv. Hosui, em Caçador-SC, na safra 2000-2001.

\begin{tabular}{cc}
\hline Época de ensacamento (dias após plena floração) e tipos de sacos \\
\hline 34 dias & 83 dias \\
\hline sem & sem \\
sem & vermelho \\
sem & marrom \\
sem & duplo \\
sem & marrom kraft comercial \\
sem & pipoca branco \\
saco pequeno & sem \\
saco pequeno & vermelho \\
saco pequeno & marrom \\
saco pequeno & duplo \\
saco pequeno & marrom kraft comercial \\
saco pequeno & pipoca branco \\
vermelho & sem \\
marrom & sem \\
duplo & sem \\
marrom kraft comercial & sem \\
pipoca branco & sem \\
\hline
\end{tabular}

O delineamento experimental foi o inteiramente casualizado, com os tratamentos arranjados no esquema fatorial $2 \times 6$, em três repetições. Os fatores testados foram o efeito da colocação de sacos pequenos de papel aos 34 dias (30-10-00) após a plena floração (ensacamento Tipo 1) e o efeito da colocação de diferentes tipos de sacos grandes de papel aos 83 dias (18-12-00) após a plena floração

\footnotetext{
${ }^{1}$ (Trabalho 151/2002). Recebido: 22/09/2002. Aceito para publicação: 30/01/2004. Trabalho financiado pelo FAP/UnC e Epagri.

${ }^{2}$ Eng.Agr.M.Sc., EPAGRI, Estação Experimental de Caçador/Universidade do Contestado, Caçador-SC, 89500-000, E-mail: faoro@ epagri.rct- sc.br

${ }^{3}$ Eng. Agr. M.Sc., EPAGRI, Estação Experimental de Caçador, Caçador-SC, 89500-000, E-mail: mmondardo@epagri.rct-sc.br
} 
(Ensacamento tipo 2). Os sacos grandes foram colocados sobre os pequenos. Adicionalmente, foram realizados mais cinco tratamentos com diferentes tipos de sacos grandes de papel apenas aos 34 dias após a plena floração (ensacamento Tipo 3), adotando o mesmo tipo de delineamento experimental. O raleio foi realizado em 24 e 25-10-00, antes do ensacamento, deixando um fruto por rácimo e espaçados em, no mínimo, $20 \mathrm{~cm}$.

Foram realizadas análises da aparência externa, peso médio, maturação e qualidade dos frutos. Para o pH do suco, açúcar e resistência da polpa, foram utilizados cinco frutos por repetição. $O$ açúcar foi medido pelo teor de Sólidos Solúveis Totais ( ${ }^{\circ}$ Brix); a firmeza (lbs) com penetrômetro manual, com ponta de $8 \mathrm{~mm}$ de diâmetro; e o pH diretamente no suco dos frutos, com pHmetro.

A aparência externa dos frutos foi avaliada conforme a seguinte escala: $1=$ aparência excelente e película lisa sem manchas; $2=$ película lisa e sem manchas, com lenticelas pouco salientes; $3=$ película com menos de 5\% de manchas de russeting e lenticelas pouco salientes; $4=$ película moderadamente áspera e lenticelas moderadamente salientes, e/ ou 5 a $15 \%$ de manchas de russeting; 5= película áspera, com lenticelas salientes, e/ou com mais de $15 \%$ de manchas. Os dados foram avaliados por estatística não-paramétrica, utilizando o teste do Friedman (quiquadrado), considerando a inexistência de variância em muitos tratamentos.

A cor de maturação do fruto foi medida pela cor de fundo, conforme a Tabela Geral de Cor (Faoro \& Shiba, 2001), a qual é composta pelas seguintes notas: $1=$ fruto imaturo, com cor de fundo verde-escura; $2=$ fruto menos imaturo, com cor de fundo verde menos escura, podendo ser destinado para armazenagem; $3=$ fruto maduro, com cor de fundo verde, apto para consumo imediato ou para armazenagem; $4 \mathrm{a}=$ fruto maduro, com cor de fundo verde-clara, apto para consumo ou para armazenagem; $4 \mathrm{~b}=$ fruto maduro, com cor de fundo verde-clara amarelada, apto somente para consumo imediato; $5 \mathrm{a}=$ fruto sobre-maduro, com cor de fundo amarelo-esverdeada clara, apto somente para consumo imediato; $5 \mathrm{~b}=$ fruto sobre-maduro, com cor de fundo amarelada, inapto para consumo; $6=$ fruto sobre-maduro, com cor de fundo amarelo-bronzeada, inapto para o consumo.

\section{RESULTADOS E DISCUSSÃO}

Em setembro e outubro de 2000, ocorreu grande quantidade de dias com chuvas ou nublados, proporcionando alta umidade, que é o principal fator para o desenvolvimento de russeting nos frutos (Gil, 1989; Basso \& Suzuki, 2001). No início do inverno, a temperatura teve pequena elevação, num curto período, voltando a declinar. O número de unidades de frio, pelo método Carolina do Norte Modificado, foi de 1.113, acima da média dos últimos 25 anos (1.074 unidades), por isso considerada satisfatória. Como resultado, a floração da 'Housui' foi uma das melhores dos últimos cinco anos. Em conseqüência, foi possível realizar um bom raleio, ficando os frutos bem distribuídos na planta.

O ensacamento não influenciou na firmeza dos frutos, no teor de sólidos solúveis totais e no peso médios dos frutos, o qual foi de $162,9 \mathrm{~g}$.

A análise de variância para a cor de maturação dos frutos mostrou efeito significativo da interação entre o ensacamento aos 34 dias e o ensacamento aos 83 dias.

Foi verificado que a maturação pouco foi afetada pela colocação de diferentes tipos de sacos grandes, aos 83 dias, quando previamente foram colocados sacos pequenos (Tabela 2). Somente os sacos vermelhos colocados sobre os sacos pequenos resultaram em menor índice de maturação, o qual não diferenciou do tratamento utilizando somente o ensacamento com sacos pequenos e com saco de pipoca branco. Este último, após as primeiras chuvas e a queda de granizo, desintegraram quase que totalmente, expondo os sacos pequenos. Pela ineficiência, portanto, não é indicado o uso de saco tipo pipoca branco e saco vermelho, pois o produtor somente estará despendendo recursos financeiros, sem vantagem alguma quanto à colheita mais precoce. Os demais tratamentos, embora tenham apresentado maior índice de maturação do fruto, não diferiram da utilização apenas de sacos pequenos aos 34 dias, concluindo não ser indicado o uso adicional de sacos grandes aos 84 dias quando se quer maior precocidade de colheita.

TABELA 2 - Cor de fundo de pêra cv. Housui na maturação, conforme Tabela Geral de Cores, em Caçador-SC, safra 2000-2001.

\begin{tabular}{lcc}
\hline \begin{tabular}{l} 
Ensacamento aos \\
\multicolumn{1}{c}{83 dias }
\end{tabular} & $\begin{array}{c}\text { Sem ensacamento } \\
\text { aos } 34 \text { dias }^{(1)}\end{array}$ & $\begin{array}{c}\text { Com ensacamento aos } \\
34 \text { dias }^{(2)}\end{array}$ \\
\hline duplo & $3,98 \mathrm{a}$ & $3,53 \mathrm{a}$ \\
marrom & $3,57 \mathrm{~b}$ & $3,53 \mathrm{a}$ \\
vermelho & $3,33 \mathrm{bc}$ & $3,13 \mathrm{~b}$ \\
kraft marrom & $3,00 \mathrm{c}$ & $3,53 \mathrm{a}$ \\
pipoca branco & $2,93 \mathrm{~cd}$ & $3,20 \mathrm{ab}$ \\
sem & $2,99 \mathrm{~d}$ & $3,33 \mathrm{ab}$ \\
\hline
\end{tabular}

Médias seguidas pela mesma letra, na coluna, não diferem estatisticamente, pelo teste de Duncan, a 5\% de probabilidade.

(1): sacos pequenos colocados aos 34 dias e sacos grandes aos 83 dias após a plena floração.

(2): somente foram colocados sacos grandes aos 83 dias após a plena floração. C. $\mathrm{V}=6,20$. Média Geral = 3,34

Já o ensacamento aos 83 dias após a plena floração, somente utilizando sacos grandes, quando os frutos estavam desenvolvidos, influenciaram na precocidade da maturação. Comparativamente, o uso de sacos duplos foi o mais eficiente, seguido pelo marrom, ambos de origem japonesa. Os frutos não ensacados tiveram o mesmo resultado que os frutos ensacados com sacos vermelhos, marrons kraft e pipoca brancos. Logo, um ensacamento tardio induz uma colheita mais precoce, sendo indicados sacos duplos ou marrons.

O ensacamento aos 34 dias, com sacos pequenos, elevou o $\mathrm{pH}$ de 4,04 para 4,29. O tipo de saco também influenciou no $\mathrm{pH}$ dos frutos (Tabela 3), sendo mais elevado utilizando o kraft marrom, seguido do duplo, do marrom e do pipoca branco. Esse aumento altera o balanço entre os sólidos solúveis totais e o $\mathrm{pH}$, podendo alterar, em consequiência, o sabor do fruto (Antunes et al., 1998; Argenta, 2002). No entanto, o fator sabor não foi avaliado nesta pesquisa.

TABELA 3 - Efeito de diferentes sacos de papel tamanho grande sobre o pH de peras cv. Housui, em Caçador-SC, safra 20002001.

$\begin{array}{cl}\text { Tipos de saco } & \mathrm{pH} \\ \text { kraft marrom } & 4,35 \mathrm{a} \\ \text { duplo } & 4,29 \mathrm{ab} \\ \text { marrom } & 4,20 \mathrm{ab} \\ \text { pipoca branco } & 4,17 \mathrm{ab} \\ \text { vermelho } & 4,07 \mathrm{bc} \\ \text { ensacamento } & 3,93 \mathrm{c}\end{array}$

Médias seguidas pela mesma letra não diferem estatisticamente, pelo teste de Duncan, a 5\% de probabilidade.

C.V=4,21 e Média Geral =4,28.

$\mathrm{Na}$ avaliação da aparência dos frutos (Tabela 4), foi verificado que, quanto antes ocorre o ensacamento, desde que utilizando sacos grandes, de coloração mais escura (marrom), simples ou duplos, melhor é a aparência externa do fruto. Quando realizado o ensacamento aos 34 dias após a plena floração, melhor resultado foi obtido também com a utilização de sacos escuros (marrons) aos 83 dias. A utilização de sacos de pipoca brancos, com ou sem ensacamento prévio de sacos pequenos, não melhorou a aparência dos frutos.

O ensacamento 34 dias após a plena floração, utilizando sacos grandes de papel duplos de cor marrom e sacos de papel kraft marrons, ou o uso de sacos pequenos aos 34 dias seguido pela colocação, aos 83 dias, dos dois tipos de sacos grandes citados anteriormente, resultaram em frutos de melhor qualidade externa (película de coloração homogênea e mais clara, lisa e com lenticelas pouco salientes). O uso de sacos 
TABELA 4 - Aparência de peras cv. Housui, após diferentes tipos de ensacamento, em Caçador-SC, safra 2000-2001.

\begin{tabular}{ccc}
\hline $\begin{array}{c}\text { Época de ensacamento (dias após plena } \\
\text { floração) e tipos de saco }\end{array}$ & $\begin{array}{c}\text { Classe de } \\
\text { aparência }\end{array}$ \\
\cline { 1 - 2 } 34 dias & 83 dias & \\
\hline duplo & sem & 2 \\
marrom kraft & sem & \\
comercial & & 3 \\
saco pequeno & marrom kraft comercial & 4 \\
marrom & sem & 4 \\
saco pequeno & duplo & 4 \\
saco pequeno & marrom & 5 \\
saco pequeno & vermelho & 5 \\
sem & marrom kraft comercial & 5 \\
sem & duplo & 5 \\
sem & marrom & 6 \\
vermelho & sem & 7 \\
sem & vermelho & 8 \\
saco pequeno & sem & 9 \\
pipoca branco & sem & 9 \\
saco pequeno & pipoca branco & 9 \\
sem & pipoca branco & 9 \\
sem & sem & \\
\hline
\end{tabular}

Nota: as classes de aparência dos tratamentos variam de 1 (melhor) a 9 (pior), conforme a separação, pelo teste de Friedman.

vermelhos e de pipoca brancos, com ou sem ensacamento prévio, não resultaram em melhoria substancial da qualidade externa do fruto.

O período mais crítico para o russeting ocorre nas primeiras fases do crescimento do fruto, ou seja, cerca de 15 a 20 dias após a plena floração (Gil S., 1989; Yuri \& Casteli, 1998). Logo, a realização do ensacamento no menor tempo possível, após a plena floração, induz melhor qualidade externa nos frutos. Assim, o uso de sacos de papel manteiga, preferencialmente parafinados e de cor escura, para melhor proteger os frutos da umidade e reduzir a incidência de luz, melhora a sua aparência.

\section{CONCLUSÕES}

1) Não há necessidade de realizar dois ensacamentos para melhorar a aparência dos frutos da cv. Housui.

2) O uso de sacos de pipoca brancos não melhora a aparência dos frutos.

3) O uso de saco de pipoca deve ser evitado devido a sua desintegração com a chuva.

4) O ensacamento dos frutos não influencia na firmeza, no teor de sólidos solúveis totais e no peso médio dos frutos.

5) $\mathrm{O} \mathrm{pH}$ da polpa aumenta com o uso de sacos duplos de papel parafinado, sacos marrons de papel manteiga parafinado, sacos de papel kraft marrons e sacos de pipoca brancos. Também sacos pequenos de papel manteiga parafinado, aos 34 dias, induzem maior $\mathrm{pH}$.

6) Ensacamento utilizando sacos duplos de papel parafinado e sacos marrons de papel manteiga parafinado tendem a proporcionar precocidade na colheita.

\section{REFERÊNCIAS BIBLIOGRÁFICAS}

ANTUNES, L.E.C.; RAMOS, J.D.; HOFFMANN, A.; SILVA, C.R.R.; CHALFUN, N.N.; PASQUAL, M. Implantação de pomares e tratos culturais especiais. Lavras: UFLA/FAEPE, 1998. 199p.

ARGENTA, L.C. Fisiologia pós-colheita: maturação, colheita e armazenagem dos frutos. In: EPAGRI. A cultura da macieira. Florianópolis: Epagri, 2002. p. 691-732.

BASSO, C.; SUZUKI, A. Distúrbios fisiológicos. In: EPAGRI. Nashi, a pêra japonesa. Florianópolis: Epagri/Jica, 2001.p. 233-242.

FAORO, I.D. Técnica e custo para o ensacamento de frutos de pêra japonesa. Revista Brasileira de Fruticultura, Jaboticabal, v.25, n.2, p. 339-340, 2003.

FAORO, I.D.; SHIBA, S. Colheita, classificação e armazenagem. In: EPAGRI. Nashi, a pêra japonesa. Florianópolis: Epagri/Jica, 2001.p. 211-231.

FAORO, I.D.; YASUNOBU, Y. Investigação dos fatores de necrose de gemas e seu controle. In: REUNIÃOTÉCNICADEAPRESENTAÇÃO DE RESULTADOS DE PESQUISA, 4., 2001, São Joaquim. Relatório anual 2000-2001.... São Joaquim: Epagri/Jica/Embrapa, 2001. p. $93-$ 94.

FAORO, I.D.; BECKER, W.F.; TSUCHIA, S. Avaliação da severidade à entomosporiose em três cultivares de pereira japonesa. In: SEMINÁRIO SOBREFRUTICULTURADECLIMATEMPERADO, 1., 2001, Florianópolis. Anais... Florianópolis: Epagri, 2001. p. 152.

GIL, G.F.S. La rugosidade o ruginosidad ("russet") de la fruta. Revista Frutícola, v. 10, n.2, p. 57-62, 1989.

NORA, I.; SUGIURA, T. Pragas da pereira. In: EPAGRI. Nashi, a pêra japonesa. Florianópolis: Epagri/Jica, 2001.p. 261-321.

YURI, J.A.; CASTELI, R. Pear russet control with giberellins and other products, in cv. Packham's Triumph. Acta Horticulturae, Wageningen, n. 475, p. 303-310, 1998. 